\title{
Über die Photolyse des Uranylformiates
}

\section{Doctoral Thesis}

Author(s):

Hatt, Emil

Publication date:

1917

Permanent link:

https://doi.org/10.3929/ethz-a-000107673

Rights / license:

In Copyright - Non-Commercial Use Permitted 


\section{Über die Photolyse des Uranylformiates}

Von der

Eidgenössischen Technischen Hochschule

in Ziirich

zur Erlangung der

Wiirde eines Doktors der technischen Wissenschaften

genehmigte

\section{Promotionsarbeit}

vorgelegt von

Emil Hatt, dipl. techn. Chemiker

aus Basel

Referent: Prof. Dr. E. Baur .

Korreferent: Prof. Dr. E. Bosshard

181

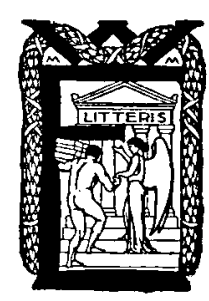

Leipzig

Verlag ron Wilhelm Engelmann

1917 


$$
\varepsilon=\frac{55 \cdot 10 \cdot 0 \cdot 9384}{0.62 \cdot 10^{-10} \cdot 7 \cdot 10^{23}}=1 \cdot 18 \cdot 10^{-11} \mathrm{erg}
$$

also ist:

$$
\frac{E}{\varepsilon}=0.4 \text {. }
$$

Als- drittes Beispiel wähle ich die Anfangsgeschwindigkeit bei mittlerer Sonnenhelligkeit.

Der Wert 0.9384 (Energie von 1 Lux in erg) wird, wie oben ${ }^{1}$ ) dargelegt, auf den vierten Teil reduziert, und wir erbalten als sekundlich einfallende Lichtenergie:

$$
500000 \cdot 10 \cdot 0 \cdot 2346 \mathrm{erg} .
$$

Es werden umgesetzt:

$109 \mathrm{ccm} 1 / 100$-norm. $K M n O_{4}$ pro Stunde, oder: ${ }^{-} \quad 1 \cdot 5 \cdot 10^{-7} \mathrm{Mol} / \mathrm{sec}=1 \cdot 5 \cdot 10^{-i} \cdot 7 \cdot 10^{23} \mathrm{Moleküle} / \mathrm{sec}$.

Demnach werden für ein Molekül verbraucht:

$$
\varepsilon=\frac{500000 \cdot 10 \cdot 0 \cdot 2346}{1 \cdot 5 \cdot 10^{-7} \cdot 7 \cdot 10^{23}}=1 \cdot 1 \mathrm{erg}
$$

also ist:

$$
\frac{E}{\varepsilon}=0.4 \text {. }
$$

Der erhaltene Quotient hat also die erwartete Grösse und ergibt insofern eine Bestätigung der Forderungen der Äquivalenzhypothese Einsteins.

\section{Zusammenfassung.}

1. Es wurde eine Methode ausgearbeitet, um Uranosalz neben Ameisensäure titrimetrisch zu bestimmen.

2. Die photochemische Zersetzung des Uranylformiats wurde be verschiedenen Konzentrationen untersucht, und es wurde festgestellt, dass das gebildete Uranosalz einen verzögernden Einfluss auf die R. G. ausübt.

3. Die photochemische Zersetzung des Uranylformiats wurde bei Beleuchtungsstärken zwischen 220 und 500000 Lux untersucht, und es wurde festgestellt, dass die Anfangsgeschwindigkeit der Beleuchtungsstärke proportional ist, während später ein geringes, aber deutliches Zurückbleiben hinter der Proportionalität mit der Lichtstärke beobachtet wird.

4. Die photochemische Zersetzung des Uranylformiats wurde bei Gegenwart verschiedener zugesetzter Substanzen untersucht, und es

1) Vgl. S. 25. 
wurde festgestellt, dass Chlorkalium, Jodkalium, Ferrichlorid, Vanadylsulfat und Vanadinsäure einen antikatalytischen Einfluss haben, während Kaliumsulfit ohne Wirkung bleibt.

5. Es wurde die oxydierende Wirkung von belichtetem Uranylsalz auf Kaliumjodid und seine reduzierende Wirkung auf Kaliumpermanganat untersucht, und durch diese Versuche ein Beweis für die Existenz einer höheren und einer niederen Oxydationsstufe in belichteten Uranylsalzlösungen erbracht. Durch diese primären Lichtprodukte werden auch die obigen Hemmungswirkungen, sowie das von E. Baur gefundene logarithmische Verbältnis zwischen Lichtstärke und Potentialverschiebung beim Becquerel-Effekt erklärt. Hierdurch erbalten die von G. Trümpler über die Natur der primären Lichtwirkung auf Uranylsalze ausgesprochenen Anschauungen weitergreifende Sicherstellung.

6. Es wurde die bei der photochemischen Zersetzung des Uranylformiates für 1 absorbiertes Energiequantum umgesetzte Anzahl Moleküle berechnet, und $\frac{h v}{\varepsilon}=0.4$ gefunden.

Die vorliegende Arbeit wurde auf Anregung und unter Leitung: von Herrn Prof. Dr. E. Baur im physikalisch-chemischen Laboratorium der Eidgenössischen Technischen Hochschule Zürich ausgeführt. Meinem hochverehrten Lehrer möchte ich auch an dieser Stelle für sein reges Interesse und seine allezeit bereitwillige Unterstützung meinen besten Dank aussprechen. 